\title{
Correction to: genetic diversity and chemical profile of Rhodophiala bifida populations from Brazil
}

\author{
Maurício Barancelli Schwedersky ${ }^{1}$ - Marina Scopel ${ }^{1} \cdot$ Luciana R. Tallini $^{1,2}$ • Jaume Bastida ${ }^{2}$. Tatiana T. Souza-Chies ${ }^{3}$. \\ Nora H. Oleas ${ }^{4}$. José Angelo Silveira Zuanazzi ${ }^{1}$
}

Published online: 4 June 2020

(C) Sociedade Brasileira de Farmacognosia 2020

\section{Correction to: Rev. Bras. Farmacogn} https://doi.org/10.1007/s43450-020-00041-5

The chemical structures appear incorrectly in the original publication of this article. They appear correctly here.<smiles>O[C@H]1CC=C2[C@@H]3C[C@H](c4cc5c(cc4CN3[C@@H]2O)OCO5)[C@@H]1O</smiles>

1

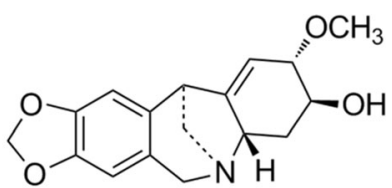

2

The online version of the original article can be found at https://doi.org/ 10.1007/s43450-020-00041-5

José Angelo Silveira Zuanazzi

zuanazzi@ufrgs.br; https://orcid.org/0000-0003-1506-6355

Maurício Barancelli Schwedersky

https://orcid.org/0000-0001-8874-4060

Marina Scopel

https://orcid.org/0000-0002-8554-8838

Luciana R. Tallini

https://orcid.org/0000-0003-3890-0971

Jaume Bastida

https://orcid.org/0000-0002-0294-5927

Tatiana T. Souza-Chies

https://orcid.org/0000-0002-1818-8748
Nora H. Oleas

https://orcid.org/0000-0002-1948-4119

1 Laboratório de Farmacognosia, Faculdade de Farmácia, Universidade Federal do Rio Grande do Sul, Porto Alegre, RS, Brazil

2 Grupo de Productos Naturales, Facultat de Farmàcia i Ciències de l'Alimentació, Universitat de Barcelona, Barcelona, Spain

3 Departamento de Botânica, Universidade Federal do Rio Grande do Sul, Porto Alegre, RS, Brazil

4 Centro de Investigación de la Biodiversidad y Cambio Climático e Ingeniería en Biodiversidad y Recursos Genéticos, Facultad de Ciencias de Medio Ambiente, Universidad Tecnológica Indoamérica, Machala y Sabanilla, Quito, Ecuador 